\title{
Diagonalization of elliptic systems via pseudodifferential projections
}

\author{
Matteo Capoferri
}

12 January 2022

\begin{abstract}
Consider an elliptic self-adjoint pseudodifferential operator $A$ acting on $m$-columns of half-densities on a closed manifold $M$, whose principal symbol is assumed to have simple eigenvalues. Relying on a basis of pseudodifferential projections commuting with $A$, we construct an almost-unitary pseudodifferential operator that diagonalizes $A$ modulo an infinitely smoothing operator. We provide an invariant algorithm for the computation of its full symbol, as well as an explicit closed formula for its subprincipal symbol. Finally, we give a quantitative description of the relation between the spectrum of $A$ and the spectrum of its approximate diagonalization, and discuss the implications at the level of spectral asymptotics.
\end{abstract}

Keywords: pseudodifferential projections, elliptic systems, unitary diagonalization, invariant subspaces, spectral asymptotics.

2020 MSC classes: primary 58J40; secondary 47A15, 35J46, 35J47, 35J48, 35P20, 58J05.

\section{Contents}

1 Introduction $\quad 2$

2 Statement of the problem $\quad 3$

3 Main results $\quad 6$

4 Construction of the operators $B_{j} \quad 9$

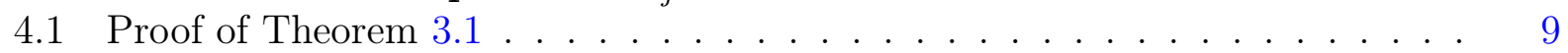

4.2 The algorithm . . . . . . . . . . . . . . . . . . . . . . . . . . . . . . . . . . . . . .

4.3 The subprincipal symbol . . . . . . . . . . . . . . . . . . 11

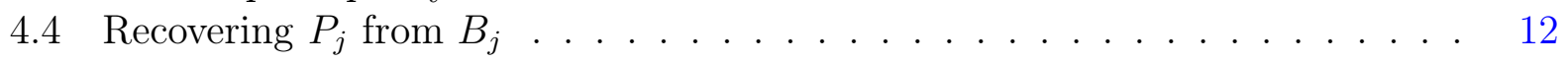

MC: School of Mathematics, Cardiff University, Senghennydd Rd, Cardiff CF24 4AG, UK; matteo.capoferri@gmail.com, http://mcapoferri.com. 
5 The diagonalized operator $\quad 13$

5.1 Action of the almost-unitary operator $B \ldots \ldots \ldots$

5.2 Spectral analysis . . . . . . . . . . . . . . . . . . . . . . . 14

5.3 Spectral asymptotics . . . . . . . . . . . . . . . . . . . . 21

6 An example: the operator of linear elasticity 24

$\begin{array}{ll}\text { Acknowledgements } & 27\end{array}$

$\begin{array}{ll}\text { Appendix A Some useful results } & 27\end{array}$

$\begin{array}{ll}\text { References } & 28\end{array}$

\section{Introduction}

Decoupling systems of (partial differential) equations is often quite useful, especially in applications, see, e.g., [24, 30, 42, 43, 44, 45]. A number of diagonalization techniques have been developed over the years, in different contexts and with varying degree of mathematical rigour. In this paper we are concerned with the diagonalization of elliptic systems of pseudodifferential equations.

In 1975 Taylor [51] showed that a first order system of pseudodifferential operators can be approximately diagonalized, as soon as one can diagonalize its principal symbol in a smooth manner. Taylor's construction establishes the existence of a diagonalizing operator by starting from the principal symbol and iteratively seeking correction terms of lower order. The matrix correction terms are prescribed to have a particular structure, so that the uniqueness of the diagonalizing operator is guaranteed, modulo an infinitely smoothing operator. An analogue of Taylor's argument in the semiclassical setting was later provided by Helffer and Sjöstrand [29].

More detailed results are available for specific operators. Let us mention a few.

In 1983 Cordes [17] performed a rigorous analysis of the Foldy-Wouthuysen transform $[24,23]$, a unitary operator that achieves the (approximate) decoupling of the positive and negative energy parts of the (massive) Dirac equation, using the tools of microlocal analysis. Unlike Taylor, Cordes does not introduce artificial constraints in his construction; as a result, the unitary operator he obtains is not uniquely defined - not even modulo an operator of order $-\infty$. In a subsequent paper [18] Cordes investigated whether one can correct the FoldyWouthuysen transform by smoothing operators so as to achieve an exact decoupling, not merely modulo smoothing. His analysis identified an obstruction to an exact diagonalization, expressed in terms of the nonvanishing of a certain 'deficiency index'.

A semiclassical Foldy-Wouthuysen transform for the Dirac operator was later constructed by Bruneau and Robert [6], following Taylor's strategy.

Nenciu and Sordoni [39] studied the approximate diagonalization and the resulting almostinvariant subspaces of multistate Klein-Gordon Hamiltonians. Their analysis was taken further by Panati, Spoh and Teufel [41] who, in the setting of adiabatic perturbation theory, considered more general Hamiltonians with 'internal' degrees of freedom. 
We should mention that diagonalization arguments feature prominently in various areas of the mathematical analysis and mathematical physics literature - see, e.g., [36, 3, 37, 52, 2]. These include approaches to (block) diagonalization that do not rely on pseudodifferential techniques, see also $[28,50,19]$.

In this paper we will approach diagonalization of elliptic pseudodifferential systems by a different route, one that is based on the theory of pseudodifferential projections, as developed by Vassiliev and the author in [13]. Our global invariant construction is different from those listed above, and will lead to an explicit algorithm for the determination of the full symbol of the almost-unitary diagonalizing operator. Being adjusted to pseudodifferential projections, our construction is particularly suitable to study the relation between the spectrum of the original operator and that of its diagonalized version. We believe that the self-contained explicit construction presented here will prove useful in applications - for example, in the context of quantum field theory in curved spacetime, compare $[4,16,5]$ and $[25,26,27,49]$ - and be more accessible for the wider mathematical physics community. From a theoretical perspective, this paper complements the analysis of invariant subspaces of elliptic systems carried out in $[13,14]$, and the spectral results therein.

\section{Statement of the problem}

Let $M$ be a closed connected manifold of dimension $d \geq 2$. We denote local coordinates on $M$ by $x=\left(x^{1}, \ldots, x^{d}\right)$ and local coordinates on the cotangent bundle $T^{*} M$ by $(x, \xi)=$ $\left(x^{1}, \ldots, x^{d}, \xi_{1}, \ldots, \xi_{d}\right)$. We denote by $T^{\prime} M:=T^{*} M \backslash\{0\}$ the cotangent bundle with the zero section removed.

Let $\mathcal{C}^{\infty}(M)$ be the vector space of smooth complex-valued half-densities over $M$ and let $\mathcal{L}^{2}(M)$ be its closure with respect to the inner product

$$
\prec f, g \succ=\int_{M} \bar{f} g d x,
$$

where $d x:=d x^{1} \ldots d x^{n}$ and the overbar denotes complex conjugation.

Given a natural number $m \geq 2$, we define $C^{\infty}(M):=\left[\mathcal{C}^{\infty}(M)\right]^{m}$ to be the vector space of $m$-columns of smooth complex-valued half-densities over $M$ and $L^{2}(M)$ to be its closure with respect to the inner product

$$
\langle u, v\rangle=\int_{M} u^{*} v d x
$$

Here and further on $*$ stands for Hermitian conjugation when applied to matrices and for adjunction when applied to operators. Of course, $L^{2}(M):=\left[\mathcal{L}^{2}(M)\right]^{m}$. The spaces $C^{\infty}(M)$ and $L^{2}(M)$ depend on the choice of $m$, but we suppress this dependence to simplify notation.

For $s \in \mathbb{R}$, we denote by $\mathcal{H}^{s}(M)$ (resp. $H^{s}(M)$ ) the usual Sobolev space, i.e. the vector space of half-densities (resp. $m$-columns of half-densities) that are in $\mathcal{L}^{2}(M)$ (resp. $L^{2}(M)$ ) together with their partial derivatives up to order $s$ for $s \geq 0$, and defined by duality for negative $s$.

Let $\Psi_{k, n}^{s}$ be the space of classical pseudodifferential operators of order $s$ with polyhomogeneous symbol, acting from $\left[\mathcal{H}^{s}(M)\right]^{k}$ to $\left[\mathcal{L}^{2}(M)\right]^{n}$. In other words, operators in $\Psi_{k, n}^{s}$ map 
$k$-columns to $n$-columns. We will adopt the notation $\Psi_{k}^{s}:=\Psi_{k, k}^{s}$. For an operator $Q \in \Psi_{k, n}^{s}$ we denote by $Q_{\text {prin }}$ and $Q_{\text {sub }}$ its principal and subprincipal symbols [21, Proposition 5.2.1], respectively.

We denote by $\operatorname{Id}_{m} \in \Psi_{m}^{0}$ the identity operator on $L^{2}(M)$, by $\mathrm{Id} \in \Psi_{1}^{0}$ the identity operator on $\mathcal{L}^{2}(M)$, and by $I$ the $m \times m$ identity matrix.

Let $A \in \Psi_{m}^{s}, s>0$, be an elliptic self-adjoint linear pseudodifferential operator, where ellipticity means that

$$
\operatorname{det} A_{\text {prin }}(x, \xi) \neq 0 \quad \forall(x, \xi) \in T^{\prime} M .
$$

Throughout this paper we assume that $A_{\text {prin }}$ has simple eigenvalues. Let $m^{+}$(resp. $m^{-}$) be the number of positive (resp. negative) eigenvalues of $A_{\text {prin. }}$. We denote by $h^{(j)}(x, \xi)$ the eigenvalues of $A_{\text {prin }}(x, \xi)$, enumerated in increasing order with positive index $j=1, \ldots, m^{+}$ for positive $h^{(j)}(x, \xi)$ and negative index $j=-1, \ldots,-m^{-}$for negative $h^{(j)}(x, \xi)$. We denote by

$$
J:=\left[-m^{-}, m^{+}\right] \cap(\mathbb{Z} \backslash\{0\})
$$

the indexing set of admissible values of $j$.

Remark 2.1. Each $h^{(j)}$ is a nowhere-vanishing smooth real-valued function on the punctured cotangent bundle $T^{\prime} M$. Furthermore, we have that $m^{+}$and $m^{-}$are constant and $m=m^{+}+m^{-}$. These facts are immediate consequences of self-adjointness, ellipticity, and connectedness of $M$.

By $v^{(j)}(x, \xi)$ we denote a normalised eigenvector of $A_{\text {prin }}(x, \xi)$ associated with the eigenvalue $h^{(j)}(x, \xi)$, and by

$$
P^{(j)}(x, \xi):=v^{(j)}(x, \xi)\left[v^{(j)}(x, \xi)\right]^{*}
$$

the corresponding eigenprojection. Throughout this paper, we assume that one can choose eigenvectors $v^{(j)}(x, \xi), j \in J$, of $A_{\text {prin }}$ smoothly for all $(x, \xi) \in T^{*} M \backslash\{0\}$.

Remark 2.2. In general, there may exist topological obstructions preventing one from being able to choose eigenvectors of the principal symbol of a matrix pseudodifferential operator in a smooth manner globally on the whole cotangent bundle, or even in the whole cotangent fibre at a single point. In the current paper, we assume that there are no such obstructions, so that one can perform a global diagonalization. Necessary and sufficient conditions for the existence of topological obstructions will be examined extensively in a separate paper [8].

Note that, for each $j, P^{(j)}(x, \xi)$ is a uniquely defined rank 1 matrix function, whereas $v^{(j)}(x, \xi)$ is only defined up to a local gauge transformation

$$
v^{(j)} \mapsto e^{i \phi^{(j)}} v^{(j)},
$$

where $\phi^{(j)}: T^{\prime} M \rightarrow \mathbb{R}$ is an arbitrary smooth function. The eigenprojections satisfy

$$
A_{\text {prin }}=\sum_{j \in J} h^{(j)} P^{(j)}, \quad \sum_{j \in J} P^{(j)}=I .
$$

The spectrum of the operator $A: H^{s}(M) \rightarrow L^{2}(M)$ is discrete and it accumulates to infinity. More precisely, if $m^{+} \geq 1$ it accumulates to $+\infty$, if $m^{-} \geq 1$ it accumulates to $-\infty$. 
Definition 2.3. We say that a symmetric pseudodifferential operator $Q \in \Psi_{m}^{s}$ is nonnegative (resp. nonpositive) modulo $\Psi_{m}^{-\infty}$ and write

$$
Q \geq 0 \bmod \Psi_{m}^{-\infty} \quad\left(\operatorname{resp} . Q \leq 0 \quad \bmod \Psi_{m}^{-\infty}\right)
$$

if there exists a symmetric operator $R \in \Psi_{m}^{-\infty}$ such that $Q+R \geq 0$ (resp. $\left.Q+R \leq 0\right)$.

In [13] the author and Vassiliev proved the following results.

Theorem 2.4. Let $A$ be as above and let $\delta$ be the Kronecker symbol.

(a) [13, Theorem 2.2] There exist $m$ pseudodifferential operators $P_{j} \in \Psi_{m}^{0}$ satisfying

(i) $\left(P_{j}\right)_{\text {prin }}=P^{(j)}$,

(ii) $P_{j}=P_{j}^{*} \bmod \Psi_{m}^{-\infty}$,

(iii) $P_{j} P_{l}=\delta_{j l} P_{j} \bmod \Psi_{m}^{-\infty}$,

(iv) $\sum_{j \in J} P_{j}=\operatorname{Id}_{m} \bmod \Psi_{m}^{-\infty}$,

(v) $\left[A, P_{j}\right]=0 \bmod \Psi_{m}^{-\infty}$.

These operators are uniquely determined, modulo $\Psi_{m}^{-\infty}$, by A.

(b) [13, Theorem 2.5] The operators $P_{j}$ from part (a) satisfy

$$
\begin{aligned}
& P_{j}^{*} A P_{j} \geq 0 \quad \bmod \Psi_{m}^{-\infty} \quad \text { for } \quad j=1, \ldots, m^{+}, \\
& P_{j}^{*} A P_{j} \leq 0 \quad \bmod \Psi_{m}^{-\infty} \quad \text { for } \quad j=-1, \ldots,-m^{-} \text {. }
\end{aligned}
$$

The operators $P_{j}$ from the above theorem form an orthonormal basis of pseudodifferential projections commuting with $A$, and an explicit algorithm for the construction of their full symbols was given in [13]. Pseudodifferential projections were exploited in [14] to partition the spectrum of $A$ into precisely $m$ infinite series of eigenvalues, singling out the contribution to the spectrum of $A$ of individual eigenvalues of $A_{\text {prin }}$ (more details about this will be recalled later on). Theorem 2.4 tells us that pseudodifferential projections allow one to decompose $A$ into $m$ sign-semidefinite operators $P_{j}^{*} A P_{j} \in \Psi_{m}^{s}$. However, these operators are not elliptic, and this was one of the major obstacles one had to overcome in [14].

We take here a different perspective on the problem and ask the following questions.

Question 1 Does there exist a pseudodifferential operator $B \in \Psi_{m}^{0}$ such that

$$
B^{*} B=\mathrm{Id}_{m} \quad \bmod \Psi_{m}^{-\infty}, \quad B B^{*}=\mathrm{Id}_{m} \quad \bmod \Psi_{m}^{-\infty},
$$

and

$$
B^{*} A B=\widetilde{A} \bmod \Psi_{m}^{-\infty},
$$

where $\widetilde{A} \in \Psi_{m}^{s}$ is a diagonal operator? 
Question 2 Assuming the answer to Question 1 is affirmative, can one exploit pseudodifferential projections to construct the operator $B$ explicitly?

A positive answer to Question 2 would yield an explicit characterisation of the diagonal operator $\widetilde{A}$. In particular, it is easy to see that the operators on the diagonal of $\widetilde{A}$ would be 'scalar' elliptic operators $a_{j} \in \Psi_{1}^{s}$ with $\left(a_{j}\right)_{\text {prin }}=h^{(j)}$. This leads to a further question.

Question 3 What is the relation between the spectrum of $A$ and the spectra of $a_{j}$, $j \in J$ ?

The goal of this paper is to provide a detailed answer to Questions 1,2 and 3 and discuss some applications.

\section{Main results}

Our main results are summarised in this section in the form of five theorems.

Theorem 3.1. For each $j \in J$ there exists a pseudodifferential operator $B_{j} \in \Psi_{1, m}^{0}$ such that

$$
\begin{gathered}
\left(B_{j}\right)_{\text {prin }}=v^{(j)}, \\
B_{j}^{*} B_{j}=\mathrm{Id} \bmod \Psi_{1}^{-\infty}, \\
P_{j} B_{j}=B_{j} \bmod \Psi_{1, m}^{-\infty} .
\end{gathered}
$$

An explicit algorithm for the calculation of the full symbol of the operators $B_{j}$ is given in subsection 4.2. Such algorithm shows that the operators $B_{j}$ are not uniquely determined by conditions (3.1)-(3.3) and characterises the degrees of freedom left in the symbol.

By carrying out the first iteration of the algorithm one obtains the following.

Theorem 3.2. The subprincipal symbol of $B_{j}$ reads

$$
\left(B_{j}\right)_{\mathrm{sub}}=\left(\frac{1}{4} \operatorname{tr}\left(\left(P_{j}\right)_{\mathrm{sub}}\right)+i f^{(j)}\right) v^{(j)}+\left(P_{j}\right)_{\mathrm{sub}} v^{(j)}+\frac{i}{2}\left\{P^{(j)}, v^{(j)}\right\},
$$

where $f^{(j)}: T^{\prime} M \rightarrow \mathbb{R}$ is an arbitrary smooth real-valued scalar function.

The curly brackets appearing in (3.4) denote the Poisson bracket

$$
\{B, C\}:=\sum_{\alpha=1}^{d}\left(B_{x^{\alpha}} C_{\xi_{\alpha}}-B_{\xi_{\alpha}} C_{x^{\alpha}}\right)
$$

on matrix-functions on the cotangent bundle. Further on in the paper we will also make use of the generalised Poisson bracket

$$
\{B, C, D\}:=\sum_{\alpha=1}^{d}\left(B_{x^{\alpha}} C D_{\xi_{\alpha}}-B_{\xi_{\alpha}} C D_{x^{\alpha}}\right) .
$$

The operators $B_{j}$ constitute the building blocks — more precisely, the 'columns' - of the sought-after almost-unitary operator $B$. 
Theorem 3.3. The operator $B \in \Psi_{m}^{0}$ defined in accordance with

$$
B: u=\left(\begin{array}{c}
u_{m^{+}} \\
\vdots \\
u_{1} \\
u_{-1} \\
\vdots \\
u_{-m^{-}}
\end{array}\right) \mapsto \sum_{j} B_{j} u_{j}
$$

satisfies conditions (2.2) and (2.3). The diagonalised operator $\widetilde{A}$ appearing in (2.3) reads

$$
\widetilde{A}=\operatorname{diag}\left(a_{m^{+}}, \ldots, a_{1}, a_{-1}, \ldots, a_{-m^{-}}\right),
$$

where

$$
a_{j}:=B_{j}^{*} A B_{j} \in \Psi_{1}^{s} .
$$

Of course, Theorem 3.3 follows easily from Theorem 3.1. The full symbols of both $B$ and the $a_{j}$ 's are explicitly determined by the full symbols of the $B_{j}$ 's.

The next two theorems establish a relation between the spectrum of our original operator $A$ and that of the $a_{j}, j \in J$, and show that the diagonalization procedure does not alter the spectrum substantially.

For a self-adjoint operator $Q$, we shall denote its spectrum by $\sigma(Q)$ and by

$$
\sigma^{+}(Q):=\sigma(Q) \cap(0,+\infty)
$$

its positive spectrum.

Let

$$
0<\lambda_{1} \leq \lambda_{2} \leq \ldots \leq \lambda_{k} \leq \ldots \rightarrow+\infty
$$

be the positive eigenvalues of $A$, enumerated with account of multiplicity. Suppose $m^{+} \geq 1$ and let

$$
0<\ell_{1}^{(j)} \leq \ell_{2}^{(j)} \leq \ldots \leq \ell_{k}^{(j)} \leq \ldots \rightarrow+\infty
$$

be the positive eigenvalues of $a_{j}, j=1, \ldots, m^{+}$, also enumerated with account of multiplicity.

Theorem 3.4. (a) We have

$$
\operatorname{dist}\left(\lambda_{k}, \bigcup_{j=1}^{m^{+}} \sigma^{+}\left(a_{j}\right)\right)=O\left(k^{-\infty}\right) \quad \text { as } \quad k \rightarrow+\infty .
$$

(b) For each $j=1, \ldots, m^{+}$we have

$$
\operatorname{dist}\left(\ell_{k}^{(j)}, \sigma^{+}(A)\right)=O\left(k^{-\infty}\right) \quad \text { as } \quad k \rightarrow+\infty .
$$

Let us combine the sequences of eigenvalues (3.9) for $j=1, \ldots, m^{+}$into a single sequence

$$
0<\zeta_{1} \leq \zeta_{2} \leq \ldots \leq \zeta_{k} \leq \ldots \rightarrow+\infty,
$$

with account of multiplicity. The sequence (3.12) coincides, up to a finite number of eigenvalues, with the positive spectrum of the operator (3.6). 
Theorem 3.5. For every $\alpha>0$ there exists $z_{\alpha} \in \mathbb{Z}$ such that

$$
\lambda_{k}=\zeta_{k+z_{\alpha}}+O\left(k^{-\alpha}\right) \quad \text { as } \quad k \rightarrow+\infty .
$$

Theorem 3.4 establishes asymptotic closeness of positive spectra of $A$ and $a_{j}, j=$ $1, \ldots, m^{+}$, whereas Theorem 3.5 establishes asymptotic closeness of individual eigenvalues, enumerated in our particular way. Note that the remainder terms in our two theorems are different. The underlying reason for this is that when proving one-to-one correspondence between eigenvalues in the limit for $k \rightarrow+\infty$ we have to take into account the potential presence of spectral clusters, i.e. eigenspaces of large multiplicity.

Theorems 3.4 and 3.5, alongside their counterpart for $j=-1, \ldots,-m^{-}$, imply a result first proved in [14], namely that the spectrum of $A$ partitions, up to an error with superpolynomial decay, into $m$ distinct series, one for each eigenvalue of $A_{\text {prin }}$. The advantage of the approach adopted in the current paper is that these series are characterised as the spectra of $m$ semibounded scalar (as opposed to non-semibounded matrix) elliptic operators, the operators $a_{j}$. In particular, Theorem 3.5 implies that one can compute the (global) Weyl coefficients of $A$ as the sum of the (global) Weyl coefficients of the $a_{j}$ 's, hence relying only on the much simpler formulae for scalar elliptic operators - see subsection 5.3.

Remark 3.6. Let us briefly discuss possible generalisations of our results. To begin with, we should point out that the ellipticity of $A$ or the fact that $A$ is of positive order are not really needed in the proofs of Theorems 3.1 and 3.2, or for the construction algorithm contained in subsection 4.2. The key assumption is the simplicity of the eigenvalues of $A_{\text {prin }}$. Moreover, our algorithm can be applied, with minimal modifications, to operators acting on more general vector bundles, e.g., to operators acting on differential forms. We do not carry out these generalisations in the current paper because doing so would hinder clarity and readability, without bringing a corresponding benefit in terms of insight. Finally, we should mention that in the presence of topological obstructions discussed in Remark 2.2 one can still pursue a local (or microlocal) diagonalization of the operator $A$ with the techniques presented in this paper. However, a diagonalization that is only local is of little or no use in spectral-theoretic applications, as will become clear in Section 5.

The paper is structured as follows.

Section 4 is devoted to the study of the operators $B_{j}$ appearing in Theorem 3.1. In subsection 4.1 we prove Theorem 3.1; in subsection 4.2 we provide a self-contained explicit algorithm for the computation of the full symbol of $B_{j}$; in subsection 4.3 we carry out the first iteration of our algorithm and obtain a closed formula for the subprincipal symbol of $B_{j}$, thus proving Theorem 3.2. We conclude the section by demonstrating, in subsection 4.4, that the pseudodifferential projection $P_{j}$ can be recovered from $B_{j}$.

Section 5 is concerned with the spectral analysis of the approximate diagonalization $\widetilde{A}$ of $A$. After briefly discussing in subsection 5.1 some basic properties of $\widetilde{A}$, in subsection 5.2 we provide our main spectral argument and prove Theorems 3.4 and 3.5. Finally, in subsection 5.3 we discuss the relation between local and global spectral asymptotics of $A$ and $\widetilde{A}$, for the special case of first order operators.

In Section 6 we apply our results to an explicit example: the operator of linear elasticity in dimension 2 . 
The paper is complemented by an appendix collecting some auxiliary results.

\section{Construction of the operators $B_{j}$}

This section is devoted to the proof of Theorems 3.1 and 3.2.

Throughout this section we will need a refined notation for the principal symbol. Namely, we denote by $(\cdot)_{\text {prin, } r}$ the principal symbol of the expression within brackets, regarded as an operator in $\Psi_{k, n}^{-r}$. The relevant values of $k$ and $n$ will be clear from the context, so we suppress the dependence on them in the notation of the refined principal symbol. We will use the refined notation whenever there is risk of confusion.

\subsection{Proof of Theorem 3.1}

For the reader's convenience, let us recall that we want to show that for each $j \in J$ there exists a pseudodifferential operator $B_{j} \in \Psi_{1, m}^{0}$ such that

$$
\begin{gathered}
\left(B_{j}\right)_{\text {prin }}=v^{(j)}, \\
B_{j}^{*} B_{j}=\mathrm{Id} \bmod \Psi_{1}^{-\infty}, \\
P_{j} B_{j}=B_{j} \bmod \Psi_{1, m}^{-\infty} .
\end{gathered}
$$

Proof. We will prove the theorem by constructing a sequence of pseudodifferential operators $B_{j, k} \in \Psi_{1, m}^{0}, k=0,1,2, \ldots$, such that

$$
\begin{gathered}
B_{j, k+1}-B_{j, k} \in \Psi_{1, m}^{-k-1}, \\
\left(B_{j, k}\right)_{\text {prin }}=v^{(j)}, \\
B_{j, k}^{*} B_{j, k}=\mathrm{Id} \bmod \Psi_{1, m}^{-k-1}, \\
P_{j} B_{j, k}=B_{j, k} \quad \bmod \Psi_{1, m}^{-k-1} .
\end{gathered}
$$

More precisely, for each $j$ we choose an arbitrary $B_{j, 0}$ satisfying $\left(B_{j, 0}\right)_{\text {prin }}=v^{(j)}$ and determine recursively pseudodifferential operators $Q_{j, k} \in \Psi_{1, m}^{-k}, k=1,2, \ldots$, such that

$$
B_{j, k}:=B_{j, k-1}+Q_{j, k}, \quad k=1,2, \ldots
$$

satisfy (4.3)-(4.4). Note that the operators $B_{j, k}$ defined in accordance with (4.5) automatically satisfy conditions (4.1) and (4.2).

Satisfying (4.3) and (4.4) reduces to solving

$$
\left(\left(B_{j, k-1}+Q_{j, k}\right)^{*}\left(B_{j, k-1}+Q_{j, k}\right)-\mathrm{Id}\right)_{\mathrm{prin}, k}=0
$$

and

$$
\left(P_{j}\left(B_{j, k-1}+Q_{j, k}\right)-B_{j, k-1}-Q_{j, k}\right)_{\operatorname{prin}, k}=0,
$$


which translate into the following system of equations for $\left(Q_{j, k}\right)_{\text {prin }}$ :

$$
\left\{\begin{array}{l}
{\left[v^{(j)}\right]^{*}\left(Q_{j, k}\right)_{\text {prin }}+\left[\left(Q_{j, k}\right)_{\text {prin }}\right]^{*} v^{(j)}=r_{j, k}} \\
\left(P^{(j)}-I\right)\left(Q_{j, k}\right)_{\text {prin }}=R_{j, k}
\end{array}\right.
$$

with

$$
r_{j, k}:=\left(\mathrm{Id}-B_{j, k-1}^{*} B_{j, k-1}\right)_{\text {prin,k }}, \quad R_{j, k}:=\left(B_{j, k-1}-P_{j} B_{j, k-1}\right)_{\text {prin }, k} .
$$

It is not hard to see that the solvability condition $P^{(j)} R_{j, k}=0$ for (4.6b) is satisfied. Indeed, we have

$$
\begin{aligned}
P^{(j)} R_{j, k} & =\left(P_{j}\right)_{\mathrm{prin}, 0}\left(B_{j, k-1}-P_{j} B_{j, k-1}\right)_{\mathrm{prin}, k} \\
& =\left(P_{j} B_{j, k-1}-P_{j} B_{j, k-1}\right)_{\mathrm{prin}, k} \\
& =0
\end{aligned}
$$

In the above calculation we used the fact that the principal symbol map is a homomorphism.

Then

$$
\begin{aligned}
\left(Q_{j, k}\right)_{\text {prin }} & =\left(\frac{1}{2} r_{j, k}+i f_{j, k}\right) v^{(j)}-R_{j, k} \\
& =\left(\frac{1}{2} r_{j, k}+i f_{j, k}\right) v^{(j)}-\sum_{l \in J \backslash\{j\}}\left(\left[v^{(l)}\right]^{*} R_{j, k}\right) v^{(l)},
\end{aligned}
$$

where $f_{j, k}: T^{\prime} M \rightarrow \mathbb{R}$ is an arbitrary smooth real valued function, solves (4.6a)-(4.7).

\subsection{The algorithm}

We reformulate the results from the previous subsection in the form of a self-contained algorithm for the construction of the full symbol of the operators $B_{j}$ and, as a straightforward consequence of (3.5), of the almost-unitary operator $B$.

Step 1. Given $m$ orthonormal eigenvectors $v^{(j)}$ of $A_{\text {prin }}$, choose $m$ arbitrary pseudodifferential operators $B_{j, 0} \in \Psi_{1, m}^{0}$ satisfying $\left(B_{j, 0}\right)_{\text {prin }}=v^{(j)}$.

Step 2. For $k=1,2, \ldots$ define

$$
B_{j, k}:=B_{j, 0}+\sum_{n=1}^{k} Q_{j, n}, \quad Q_{j, n} \in \Psi_{1, m}^{-n} .
$$

Assuming we have determined the pseudodifferential operator $B_{j, k-1}$, compute the following quantities:

(i) $r_{j, k}:=\left(\mathrm{Id}-B_{j, k-1}^{*} B_{j, k-1}\right)_{\operatorname{prin}, k}$,

(ii) $R_{j, k}:=\left(B_{j, k-1}-P_{j} B_{j, k-1}\right)_{\operatorname{prin}, k}$. 
Step 3. Choose a pseudodifferential operator $Q_{j, k} \in \Psi_{1, m}^{-k}$ satisfying

$$
\left(Q_{j, k}\right)_{\text {prin }}=\left(\frac{1}{2} r_{j, k}+i f_{j, k}\right) v^{(j)}-R_{j, k},
$$

where $f_{j, k}: T^{\prime} M \rightarrow \mathbb{R}$ is an arbitrary smooth real-valued function.

Step 4. Put

$$
B_{j} \sim B_{j, 0}+\sum_{k=1}^{+\infty} Q_{j, k}
$$

Here $\sim$ stands for asymptotic expansion in smoothness, namely,

$$
B_{j}-\left(B_{j, 0}+\sum_{k=1}^{n} Q_{j, k}\right) \in \Psi_{1, m}^{-n-1} .
$$

Remark 4.1. It transpires from the above algorithm that there are two different sources for the non-uniqueness of $B$ :

(i) the eigenvectors $v^{(j)}$ are only defined up to a gauge transformation (2.1);

(ii) once we have fixed the eigenvectors $v^{(j)}$, for each $B_{j}$ at every stage of the iterative process we have one real-valued degree of freedom, see (4.9).

This agrees with what observed, in a somewhat different setting, by Panati, Spohn and Teufel [41]. We should mention that great care is needed when dealing with the gauge transformation (2.1): failing to properly account for (2.1) and the curvature it brings about led to mistakes in spectral-theoretic publications, see [15, Sec. 11]. See also [40, 22, 10, 34] for a wider picture on the role of gauge transformations in the analysis of systems of PDEs.

\subsection{The subprincipal symbol}

Let us carry out the first iteration of our algorithm to obtain a formula for the subprincipal symbol of $B_{j}$, thus proving Theorem 3.2.

Without loss of generality, let us choose an operator $B_{j, 0} \in \Psi_{1, m}^{0}$ satisfying $\left(B_{j, 0}\right)_{\text {prin }}=v^{(j)}$ and the additional property

$$
\left(B_{j, 0}\right)_{\mathrm{sub}}=0
$$

Recall that the subprincipal symbol of the composition of two pseudodifferential operators is given by

$$
(C D)_{\text {sub }}=C_{\text {prin }} D_{\text {sub }}+C_{\text {sub }} D_{\text {prin }}+\frac{i}{2}\left\{C_{\text {prin }}, D_{\text {prin }}\right\},
$$

see [20, Eqn (1.4)] where the opposite convention for the sign of the Poisson brackets is adopted. 
On account of (4.11), (4.12), (3.1) and the fact that $\operatorname{Id}_{\text {sub }}=0$, we have

$$
\begin{aligned}
r_{j, 1} & =\left(\operatorname{Id}-B_{j, 0}^{*} B_{j, 0}\right)_{\text {sub }} \\
& =-\left(\left[v^{(j)}\right]^{*}\left(B_{j, 0}\right)_{\text {sub }}+\left[\left(B_{j, 0}\right)_{\text {sub }}\right]^{*} v^{(j)}+\frac{i}{2}\left\{\left[v^{(j)}\right]^{*}, v^{(j)}\right\}\right) \\
& =\frac{1}{2} \operatorname{tr}\left(\left(P_{j}\right)_{\text {sub }}\right) .
\end{aligned}
$$

In the last step of the above calculation we resorted to the identity

$$
\left\{\left[v^{(j)}\right]^{*}, v^{(j)}\right\}=i \operatorname{tr}\left(\left(P_{j}\right)_{\mathrm{sub}}\right),
$$

which is obtained by taking the trace in [13, Theorem 2.3] or by combining [15, Eqn. (1.20)] with [14, Corollary 4.2].

Similarly, in view of (4.11) and (4.12) we get

$$
\begin{aligned}
R_{j, 1} & =\left(B_{j, 0}-P_{j} B_{j, 0}\right)_{\mathrm{sub}} \\
& =-\left(P^{(j)}\left(B_{j, 0}\right)_{\mathrm{sub}}+\left(P_{j}\right)_{\mathrm{sub}} v^{(j)}+\frac{i}{2}\left\{P^{(j)}, v^{(j)}\right\}\right) \\
& =-\left(\left(P_{j}\right)_{\mathrm{sub}} v^{(j)}+\frac{i}{2}\left\{P^{(j)}, v^{(j)}\right\}\right) .
\end{aligned}
$$

Substituting (4.13) and (4.14) into (4.9) we arrive at (3.4).

Remark 4.2. It is not hard to check directly from $(4.14)$ that $\left[v^{(j)}\right]^{*} R_{j, 1}=0$. Indeed, $[13$, Theorem 2.3] tells us that

$$
\left[v^{(j)}\right]^{*}\left(P_{j}\right)_{\mathrm{sub}} v^{(j)}=-\frac{i}{2}\left[v^{(j)}\right]^{*}\left\{P^{(j)}, P^{(j)}\right\} v^{(j)},
$$

whereas a direct calculation exploiting properties of Poisson brackets gives us

$$
\begin{aligned}
\frac{i}{2}\left[v^{(j)}\right]^{*}\left\{P^{(j)}, v^{(j)}\right\} & =\frac{i}{2}\left[v^{(j)}\right]^{*}\left\{P^{(j)}, P^{(j)} v^{(j)}\right\} \\
& =\frac{i}{2}\left[v^{(j)}\right]^{*}\left(\left\{P^{(j)}, P^{(j)}, v^{(j)}\right\}+\left\{P^{(j)}, P^{(j)}\right\} v^{(j)}\right) \\
& =\frac{i}{2}\left[v^{(j)}\right]^{*}\left(\left\{\left(P^{(j)}\right)^{2}, v^{(j)}\right\}-P^{(j)}\left\{P^{(j)}, v^{(j)}\right\}+\left\{P^{(j)}, P^{(j)}\right\} v^{(j)}\right) \\
& =\frac{i}{2}\left[v^{(j)}\right]^{*}\left\{P^{(j)}, P^{(j)}\right\} v^{(j)},
\end{aligned}
$$

which is the same as (4.15), but with opposite sign.

\subsection{Recovering $P_{j}$ from $B_{j}$}

Before moving on to the spectral analysis, let us prove an identity relating the operators $B_{j}$ and pseudodifferential projections $P_{j}$, which will be quite useful later on. 
Proposition 4.3. We have

$$
B_{j} B_{j}^{*}=P_{j} \quad \bmod \Psi_{m}^{-\infty}, \quad j \in J,
$$

and

$$
\sum_{j \in J} B_{j} B_{j}^{*}=\operatorname{Id}_{m} \quad \bmod \Psi_{m}^{-\infty}
$$

Proof. Let us define

$$
S_{j}:=B_{j} B_{j}^{*}-P_{j} .
$$

Arguing by contradiction, suppose that

$$
S_{j} \in \Psi_{m}^{-k} \quad \text { but } \quad S_{j} \notin \Psi_{m}^{-k-1} .
$$

This means that

$$
\left(S_{j}\right)_{\operatorname{prin}, k}(x, \xi) \neq 0 \quad \text { for some } \quad(x, \xi) \in T^{\prime} M .
$$

Now, formula (3.3) and Theorem 2.4(a) imply

$$
S_{j} \sum_{l \in J} P_{l}=0 \bmod \Psi_{m}^{-\infty}
$$

The latter, in particular, implies

$$
\left(S_{j} \sum_{l \in J} P_{l}\right)_{\text {prin }, k}=0
$$

But

$$
\left(S_{j} \sum_{l \in J} P_{l}\right)_{\operatorname{prin}, k}=\left(S_{j}\right)_{\operatorname{prin}, k}\left(\sum_{l \in J} P_{l}\right)_{\text {prin }, 0}=\left(S_{j}\right)_{\operatorname{prin}, k} .
$$

Formulae (4.19) and (4.20) contradict (4.18), so we have proved (4.16).

Formula (4.17) now follows from (4.16) and Theorem 2.4.

\section{The diagonalized operator}

\subsection{Action of the almost-unitary operator $B$}

Define the matrix operator

$$
B:=\left(B_{m^{+}}, \ldots, B_{1}, B_{-1}, \ldots, B_{m^{-1}}\right) .
$$

Of course, $B \in \Psi_{m}^{0}$ and its action on $L^{2}(M)$ is given by (3.5). Let us label rows and columns of matrix operators from $\Psi_{m}^{r}, r \in \mathbb{R}$, by means of the elements of $J$, listed in decreasing order from $m^{+}$to $m^{-}$, so that matrix elements of $B$ read

$$
(B)_{p q}=\left(B_{q}\right)_{p}, \quad p, q \in J
$$


It is easy to see that the adjoint $B^{*} \in \Psi_{m}^{0}$ of $B$ is given by

$$
B^{*}: f \mapsto\left(\begin{array}{c}
B_{m^{+}}^{*} f \\
\vdots \\
B_{1}^{*} f \\
B_{-1}^{*} f \\
\vdots \\
B_{-m^{-}}^{*} f
\end{array}\right), \quad\left(B^{*} f\right)_{p}=B_{p}^{*} f .
$$

Formulae (3.2), (3.3) and Theorem 2.4 immediately imply

$$
B^{*} B=\operatorname{Id}_{m} \bmod \Psi_{m}^{-\infty} .
$$

Proposition 4.3 and Theorem 2.4 imply

$$
B B^{*}=\operatorname{Id}_{m} \bmod \Psi_{m}^{-\infty} .
$$

In view of (5.3) and (5.4) we conclude that $B$ is almost-unitary, up to an infinitely smoothing operator.

Formulae (3.2) and (3.3), combined with Theorem 2.4, yield

$$
\begin{aligned}
\left(B^{*} A B\right)_{l j} & =B_{l}^{*} A B_{j}=B_{l}^{*} P_{l} A P_{j} B_{j} \quad \bmod \Psi_{1}^{-\infty}=B_{l}^{*} A P_{l} P_{j} B_{j} \quad \bmod \Psi_{1}^{-\infty} \\
& =\delta_{l j} B_{j}^{*} A B_{j} \quad \bmod \Psi_{1}^{-\infty},
\end{aligned}
$$

namely, $\widetilde{A}:=B^{*} A B$ is a diagonal operator modulo $\Psi_{m}^{-\infty}$. The operators appearing on the diagonal are elliptic self-adjoint pseudodifferential operators of order $s$ from $\mathcal{H}^{s}(M)$ to $\mathcal{L}^{2}(M)$ given by

$$
a_{j}:=B_{j}^{*} A B_{j}, \quad\left(a_{j}\right)_{\text {prin }}=h^{(j)}, \quad j \in J .
$$

Hence, we have obtained Theorem 3.3.

Remark 5.1. Observe that one can easily compute the subprincipal symbol of $\widetilde{A}$ via (5.5), (3.4) and the identity

$$
\begin{aligned}
(P Q R)_{\mathrm{sub}}=P_{\mathrm{sub}} Q_{\text {prin }} R_{\text {prin }}+P_{\text {prin }} Q_{\mathrm{sub}} R_{\text {prin }} & +P_{\text {prin }} Q_{\text {prin }} R_{\text {sub }} \\
& +\frac{i}{2}(\{P, Q\} R+\{P, Q, R\}+P\{Q, R\}) .
\end{aligned}
$$

In fact, (5.5) yields, on account of subsection 4.2, an explicit algorithm for the computation of the full symbol of $\widetilde{A}$.

\subsection{Spectral analysis}

In order to prove Theorem 3.4 and Theorem 3.5, we will rely on a strategy developed by the author and Vassiliev in [14]. Throughout this section, all estimates are to be understood as asymptotic estimates as $k \rightarrow+\infty$, unless otherwise stated. 
Let us introduce the auxiliary operators

$$
A_{j}:=P_{j}^{*} A P_{j}-\operatorname{sgn}(j) \sum_{l \neq j} \operatorname{sgn}(l) P_{l}^{*} A P_{l}, \quad j \in J .
$$

Here and further on sgn returns the sign the nonzero integer number it acts upon. The operators $A_{j} \in \Psi_{m}^{s}$ are elliptic and coincide, for $j>0$ and modulo $\Psi_{m}^{-\infty}$, with the operators $A_{j}$ introduced in $\left[14\right.$, Sec. 2]. Observe that $\left(A_{j}\right)_{\text {prin }}$ has precisely one positive (resp. negative) eigenvalue for positive (resp. negative) $j$ - the eigenvalue $h^{(j)}$.

Conjugating $A_{j}$ by $B$ we get

$$
\begin{aligned}
& \left(B^{*} A_{j} B\right)_{l r}=\delta_{l r} \delta_{l j} a_{j}-\operatorname{sgn}(j \cdot l) \delta_{l r}\left(1-\delta_{l j}\right) a_{l} \bmod \Psi_{1}^{-\infty},
\end{aligned}
$$

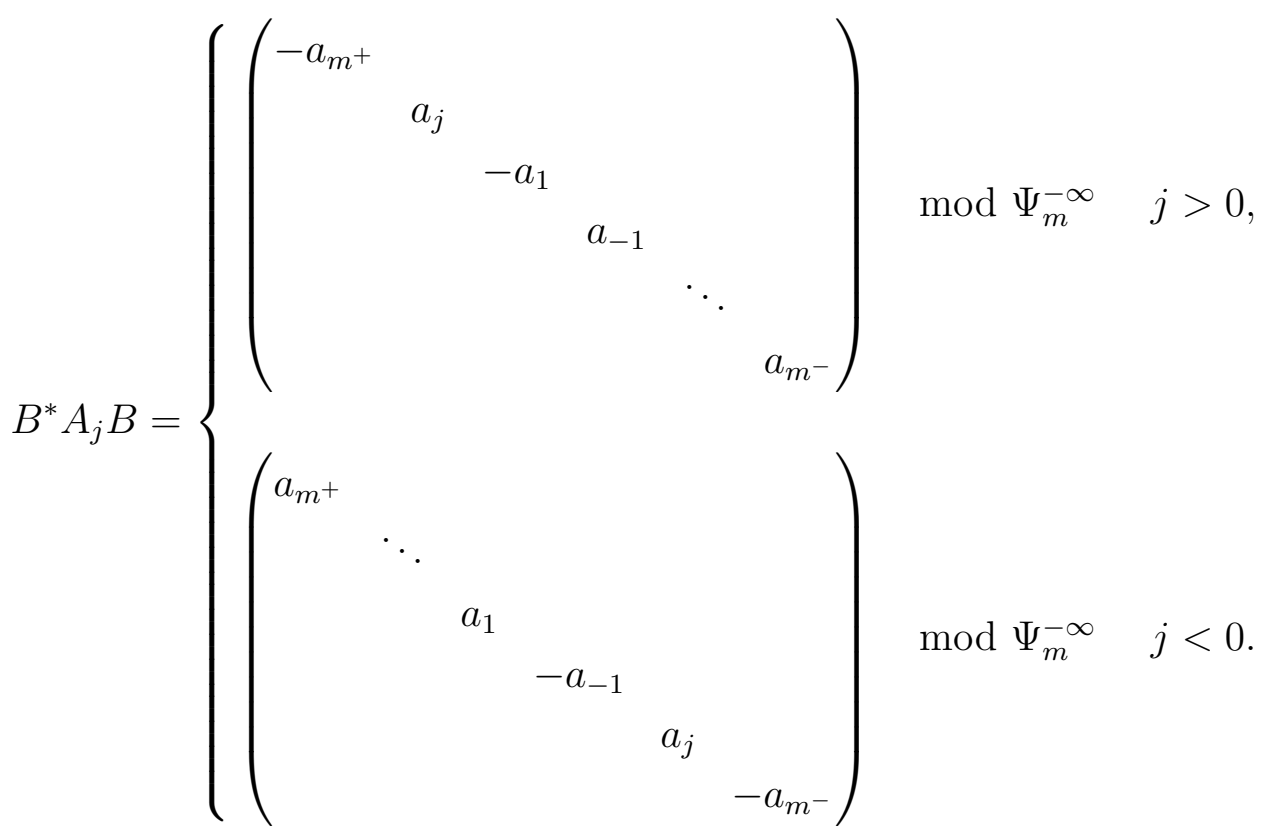

Suppose $j>0$. Then (5.8) suggests that the positive spectrum of $A_{j}$ is asymptotically described by the spectrum of $a_{j}$ and vice versa. In the remainder of this section we will provide a rigorous proof for this, accounting for the fact that all the above identities are only modulo smoothing operators. The latter will result in the appearance of remainder terms.

Our strategy goes as follows. First, we relate the spectra of $a_{j}, j \in J$, with the spectra of $A_{j}, j \in J$. Then, we exploit the results on the relation between the spectra of $A_{j}, j \in J$, and the spectrum of $A$ obtained in [14] to establish a relation between the spectra of $a_{j}, j \in J$, and the spectrum of $A$.

Remark 5.2. We should like to point out that one can prove Theorems 3.4 and 3.5 directly, without the need to introduce the operators $A_{j}$ and rely on results from [14]. As this would make the proof substantially longer without bringing about an equivalent benefit in terms of insight, we decided against going down this path. Moreover, the operators $A_{j}$ are, generally speaking, more suitable to study refined spectral properties of $A$ - see subsection 5.3; relating the spectra of $A_{j}$ and $a_{j}$ contributes some additional information which may be useful in future works. 
Without loss of generality, we will prove our results for $j>0$. The case $j<0$ is similar, and the corresponding proof follows from the case $j>0$ by replacing $A$ with $-A$.

Let

$$
0<\lambda_{1}^{(j)} \leq \lambda_{2}^{(j)} \leq \ldots \leq \lambda_{k}^{(j)} \leq \ldots \rightarrow+\infty
$$

be the positive eigenvalues of $A_{j}$, enumerated with account of multiplicity.

Notation 5.3. When we write

$$
f=O\left(k^{-\infty}\right),
$$

the estimate is to be understood as follows: any given partial derivative of the quantity on the LHS is estimated by any given negative power of $k$ uniformly over $M$.

Theorem 5.4. For $j \in J, j>0$, we have

(i) $\operatorname{dist}\left(\ell_{k}^{(j)}, \sigma^{+}\left(A_{j}\right)\right)=O\left(k^{-\infty}\right)$ as $k \rightarrow+\infty$,

(ii) $\operatorname{dist}\left(\lambda_{k}^{(j)}, \sigma^{+}\left(a_{j}\right)\right)=O\left(k^{-\infty}\right) \quad$ as $k \rightarrow+\infty$,

where $\ell_{k}^{(j)}$ and $\lambda_{k}^{(j)}$ are defined in accordance with (3.9) and (5.9), respectively.

Proof. (i) Let $f_{k}^{(j)}$ be a normalised eigenfunction of $a_{j}$ corresponding to the eigenvalue $\ell_{k}^{(j)}$,

$$
a_{j} f_{k}^{(j)}=\ell_{k}^{(j)} f_{k}^{(j)}, \quad\left\|f_{k}^{(j)}\right\|_{\mathcal{L}^{2}}=1 .
$$

For every $r=1,2, \ldots$ and every $Q \in \Psi_{1, r}^{-\infty}$, we have

$$
Q f_{k}^{(j)}=O\left(k^{-\infty}\right)
$$

Formula (5.11) follows from Weyl's Law [14, Theorem B.1]

$$
\ell_{k}^{(j)}=c k^{s / d}+o\left(k^{s / d}\right)
$$

(recall that $A$ is an operator of order $s$ ) and the identity

$$
Q f_{k}^{(j)}=\left(\ell_{k}^{(j)}\right)^{-n} Q\left(a_{j}\right)^{n} f_{k}^{(j)} \quad \forall n \in \mathbb{N} .
$$

Put $v_{k}^{(j)}:=B_{j} f_{k}^{(j)}$. We claim that

$$
A_{j} v_{k}^{(j)}=\ell_{k}^{(j)} v_{k}^{(j)}+O\left(k^{-\infty}\right) .
$$

Indeed, taking into account (5.7), (5.11) and (3.3), for $l \in J \backslash\{j\}$ we have

$$
B_{l}^{*} A_{j} v_{k}^{(j)}=B_{l}^{*} P_{l}^{*} A_{j} P_{j} v_{k}^{(j)}+O\left(k^{-\infty}\right)=O\left(k^{-\infty}\right) .
$$

Hence, (4.17) implies

$$
A_{j} v_{k}^{(j)}=B_{j} B_{j}^{*} A_{j} v_{k}^{(j)}+O\left(k^{-\infty}\right),
$$

which, combined with (5.10), (5.11) and the definition of $v_{k}^{(j)}$, yields (5.12). 
Formulae (5.12), (5.10) and the estimate

$$
\left\|v_{k}^{(j)}\right\|_{L^{2}}^{2}=\left\langle B_{j} f_{k}^{(j)}, B_{j} f_{k}^{(j)}\right\rangle=\prec B_{j}^{*} B_{j} f_{k}^{(j)}, f_{k}^{(j)} \succ=\left\|f_{k}^{(j)}\right\|_{\mathcal{L}^{2}}^{2}+O\left(k^{-\infty}\right)
$$

(recall (3.2)) give us (i).

(ii) Let $v_{k}^{(j)}$ be a normalised eigenfunction of $A_{j}$ corresponding to the eigenvalue $\lambda_{k}^{(j)}$,

$$
A_{j} v_{k}^{(j)}=\lambda_{k}^{(j)} v_{k}^{(j)}, \quad\left\|v_{k}^{(j)}\right\|_{L^{2}}=1 .
$$

Arguing as in part (i), one obtains that, for every $r=1,2, \ldots$ and every $Q \in \Psi_{m, r}^{-\infty}$,

$$
Q v_{k}^{(j)}=O\left(k^{-\infty}\right) .
$$

It was shown in [14, Proposition 3.1] that

$$
P_{l} v_{k}^{(j)}=O\left(k^{-\infty}\right) \text { for } \quad l \in J \backslash\{j\} .
$$

The latter, alongside Proposition 4.3 and (5.14), implies

$$
B_{l} B_{l}^{*} v_{k}^{(j)}=O\left(k^{-\infty}\right) \quad \text { for } \quad l \in J \backslash\{j\}
$$

and

$$
v_{k}^{(j)}=B_{j} B_{j}^{*} v_{k}^{(j)}+O\left(k^{-\infty}\right) .
$$

Combining (5.13) and (5.15), and using once again (5.14), one obtains

$$
A_{j} B_{j} B_{j}^{*} v_{k}^{(j)}=\lambda_{k}^{(j)} B_{j} B_{j}^{*} v_{k}^{(j)}+O\left(k^{-\infty}\right) .
$$

Composing the above equation with $B_{j}^{*}$ on the left, and using (3.2) and (5.14), we arrive at

$$
a_{j} B_{j}^{*} v_{k}^{(j)}=\lambda_{k}^{(j)} B_{j}^{*} v_{k}^{(j)}+O\left(k^{-\infty}\right) .
$$

As, on account of (5.15), we have

$$
\left\|B_{j}^{*} v_{k}^{(j)}\right\|_{\mathcal{L}^{2}}=\left\|v_{k}^{(j)}\right\|_{L^{2}}+O\left(k^{-\infty}\right)
$$

formulae (5.13) and (5.16) give us (ii).

Theorem 5.4 establishes closeness of the spectra of $a_{j}$ and $A_{j}$. The following theorem goes a step further and establishes closeness of individual eigenvalues.

Theorem 5.5. For every $\alpha>0$ there exists $z_{\alpha} \in \mathbb{Z}$ such that

$$
\ell_{k}^{(j)}=\lambda_{k+z_{\alpha}}^{(j)}+O\left(k^{-\alpha}\right) \quad \forall j=1, \ldots, m^{+} .
$$




$$
\sim \nu_{n}^{-\frac{\alpha d}{s}}
$$

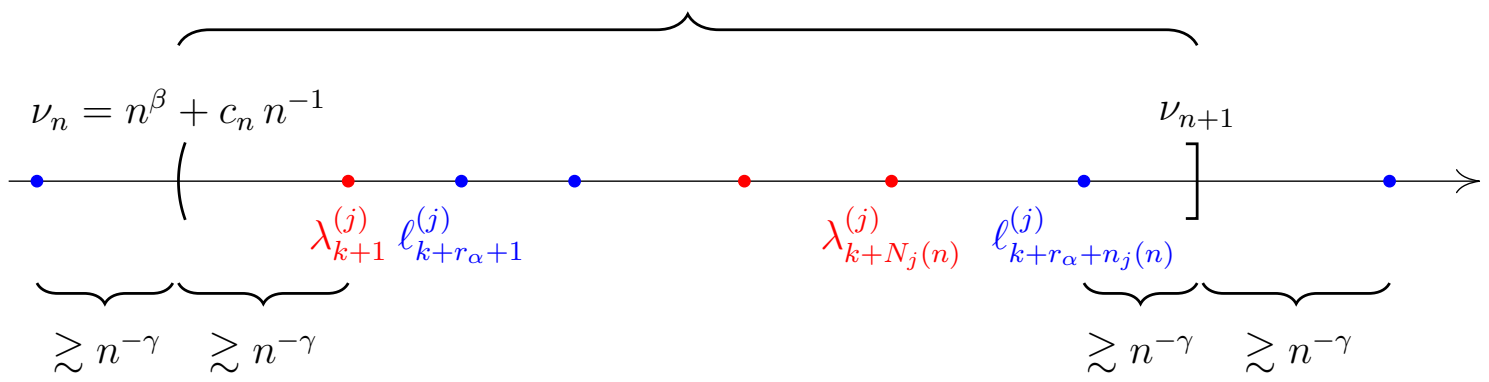

Figure 1: Partition of the positive real line

Proof. Let us partition the positive real line $(0,+\infty)$ into intervals $\left(\nu_{n}, \nu_{n+1}\right], n=0,1,2, \ldots$, whose endpoints are defined in accordance with

$$
\nu_{0}=0, \quad \nu_{n}:=n^{\beta}+c_{n} n^{-1}, \quad n=1,2, \ldots,
$$

where

$$
\beta:=\frac{1}{1+\frac{\alpha d}{s}}
$$

and the constants $c_{n} \in[-\beta / 4, \beta / 4]$ are chosen in such a way that

$$
\min _{j=1, \ldots, m^{+}} \min \left\{\operatorname{dist}\left(\nu_{n}, \sigma^{+}\left(a_{j}\right)\right), \operatorname{dist}\left(\nu_{n}, \sigma^{+}\left(A_{j}\right)\right)\right\} \geq C n^{-\gamma}
$$

for some constant $C$ and

$$
\gamma:=1+\frac{d \beta}{s} .
$$

That such a partition exists follows in a straightforward manner from the results in [14, Subsec. 3.2.1].

A simple asymptotic estimate tells us that

$$
\nu_{n+1}-\nu_{n}=O\left(\nu_{n}^{-\frac{\alpha d}{s}}\right) \quad \text { as } \quad n \rightarrow+\infty .
$$

Our strategy consists in proving that, asymptotically, each interval $\left(\nu_{n}, \nu_{n+1}\right]$ contains the same number of eigenvalues $\ell_{j}^{(j)}$ and $\lambda_{k}^{(j)}$, and then using (5.19) to achieve (5.17) with the desired accuracy — see Figure 1. To this end, let us define

$$
\begin{gathered}
N_{j}(n):=\#\left\{\lambda_{k}^{(j)}: \nu_{n} \leq \lambda_{k}^{(j)} \leq \nu_{n+1}\right\}, \\
n_{j}(n):=\#\left\{\ell_{k}^{(j)}: \nu_{n} \leq \ell_{k}^{(j)} \leq \nu_{n+1}\right\} .
\end{gathered}
$$

Let us begin by showing that for sufficiently large $n$ we have

$$
N_{j}(n) \leq n_{j}(n) .
$$


Suppose $N_{j}(n)=r$. Let $\lambda_{q+1}^{(j)}, \leq \ldots, \leq \lambda_{q+r}^{(j)}$ be the $r$ eigenvalues of $A_{j}$ in $\left(\nu_{n}, \nu_{n+1}\right.$ ) (recall that there is a neighbourhood of the endpoints free from eigenvalues) and let $v_{1}, \ldots, v_{r}$ be the corresponding orthonormalised eigenfunctions,

$$
\left\langle v_{k}, v_{k^{\prime}}\right\rangle=\delta_{k k^{\prime}}, \quad k, k^{\prime}=1, \ldots, r .
$$

It follows from (5.15) that

$$
\prec B_{j}^{*} v_{k}, B_{j}^{*} v_{k^{\prime}} \succ=\delta_{k k^{\prime}}+O\left(n^{-\infty}\right) \quad \text { as } \quad n \rightarrow+\infty .
$$

Let $F$ be the $r \times r$ matrix whose matrix elements are given by

$$
F_{k k^{\prime}}:=\prec B_{j}^{*} v_{k}, B_{j}^{*} v_{k^{\prime}} \succ, \quad k, k^{\prime}=1, \ldots, r,
$$

and let $G$ be the matrix given by Lemma A.2. Define

$$
f_{k}:=\sum_{l=1}^{r} G_{l k} B_{j}^{*} v_{l}
$$

Then formula (A.2) implies

$$
\prec f_{k}, f_{k^{\prime}} \succ=\delta_{k k^{\prime}}, \quad k, k^{\prime}=1, \ldots, r,
$$

whereas formulae (A.3), (5.21) and elliptic regularity theory imply

$$
f_{k}=B_{j}^{*} v_{k}+O\left(n^{-\infty}\right) \quad \text { as } n \rightarrow+\infty .
$$

Observe that one cannot achieve (5.23)-(5.24) via the Gram-Schmidt process, because the latter involves a number of terms growing factorially with $r$, which would produce too big an error in (5.24).

Combining (5.24) and (5.16) we conclude that for every $\widetilde{\gamma}>0$ there exists a constant $C_{\tilde{\gamma}}$ such that

$$
\left\|\left(a_{j}-\lambda_{q+k}^{(j)}\right) f_{k}\right\|_{\mathcal{L}^{2}} \leq C_{\widetilde{\gamma}} n^{-\widetilde{\gamma}}, \quad k=1, \ldots, r .
$$

In view of (5.25) and (5.23) we can apply Lemma A.1 to get

$$
\#\left\{\ell_{k}^{(j)} \mid \ell_{k}^{(j)} \in\left[\lambda_{q+1}^{(j)}-C_{\tilde{\gamma}} \sqrt{r} n^{-\widetilde{\gamma}}, \lambda_{q+r}^{(j)}+C_{\tilde{\gamma}} \sqrt{r} n^{-\widetilde{\gamma}}\right]\right\} \geq r .
$$

Now, Weyl's law tells us that $r=O\left(n^{\beta d / s}\right)$. Therefore, if $\widetilde{\gamma}>\frac{\beta d}{2 s}+\gamma$, condition (5.18) implies that for sufficiently large $n$ we have

$$
\left[\lambda_{q+1}^{(j)}-C_{\widetilde{\gamma}} \sqrt{r} n^{-\widetilde{\gamma}}, \lambda_{q+r}^{(j)}+C_{\widetilde{\gamma}} \sqrt{r} n^{-\widetilde{\gamma}}\right] \subset\left(\nu_{n}, \nu_{n+1}\right) .
$$

Formulae (5.26) and (5.27) give us (5.20).

Next, let us show that for sufficiently large $n$ we have

$$
n_{j}(n) \leq N_{j}(n) .
$$


Suppose $n_{j}(n)=r$. Let $\ell_{q+1}^{(j)}, \leq \ldots, \leq \ell_{q+r}^{(j)}$ be the $r$ eigenvalues of $a_{j}$ in $\left(\nu_{n}, \nu_{n+1}\right]$ (recall that there is a neighbourhood of the endpoints free from eigenvalues) and let $f_{1}, \ldots, f_{r}$ be the corresponding orthonormalised eigenfunctions,

$$
\left\langle f_{k}, f_{k^{\prime}}\right\rangle=\delta_{k k^{\prime}}, \quad k, k^{\prime}=1, \ldots, r .
$$

Conditions (3.2)-(3.3), Theorem 2.4 and (5.29) imply

$$
\left\langle B_{j} f_{k}, B_{j} f_{k^{\prime}}\right\rangle=\delta_{k k^{\prime}}+O\left(n^{-\infty}\right) \text { as } n \rightarrow+\infty .
$$

Let $F$ be the matrix whose matrix elements are given by

$$
F_{k k^{\prime}}:=\left\langle B_{j} f_{k}, B_{j} f_{k^{\prime}}\right\rangle, \quad k, k^{\prime}=1, \ldots, r,
$$

and let $G$ be the matrix given by Lemma A.2. Arguing as above and using (5.12), we conclude that the $m$-columns

$$
v_{k}:=\sum_{l=1}^{r} G_{l k} B_{j} f_{l}, \quad k=1, \ldots, r
$$

satisfy

$$
\left\langle v_{k}, v_{k^{\prime}}\right\rangle=\delta_{k k^{\prime}}, \quad k, k^{\prime}=1, \ldots, r,
$$

and for every $\widetilde{\gamma}>0$ there exists $C_{\widetilde{\gamma}}>0$ such that

$$
\left\|\left(A_{j}-\ell_{q+k}^{(j)}\right) v_{k}\right\|_{L^{2}} \leq C_{\widetilde{\gamma}} n^{-\widetilde{\gamma}} .
$$

Using once again Lemma A.1 one obtains analogues of (5.26) and (5.27), which in turn imply (5.28).

All in all, on account of (5.20) and (5.28), we have established that there exists $K \in \mathbb{N}$ such that for all $n>K$ we have

$$
N_{j}(n)=n_{j}(n) .
$$

Suppose $\lambda_{k}^{(j)} \in\left(\nu_{n}, \nu_{n+1}\right]$. Then the Weyl's Law gives us

$$
\nu_{n}=c k^{\frac{s}{d}}+o\left(k^{\frac{s}{d}}\right)
$$

where $c$ is a constant that can be explicitly computed. We can use (5.31) to equivalently recast (5.19) as

$$
\nu_{n+1}-\nu_{n}=O\left(k^{-\alpha}\right) .
$$

Combining formulae (5.30) and (5.32) we arrive at (5.17)

Remark 5.6. Observe that Theorem 5.4 follows from Theorem 5.5; we stated the two theorems as separate results for the sake of logical clarity. Also note that the additional material is not completely unnecessary: intermediate results obtained in the proof of the former theorem are needed in the proof of the latter.

We are now in a position to prove Theorems 3.4 and 3.5.

Proof of Theorem 3.4. On account of (5.7) and [14, Eqn. (2.2)], Theorem 5.4(ii) and [14, Theorem 2.2] imply (3.10), whereas Theorem 5.4(i) and [14, Theorem 2.1] imply (3.11).

Proof of Theorem 3.5. On account of (5.7) and [14, Eqn. (2.2)], formula (3.13) follows from Theorem 5.5 and [14, Theorem 2.3]. 


\subsection{Spectral asymptotics}

In this section we will discuss the relation between the Weyl coefficients of $A$ and those of its diagonalised version $\widetilde{A}$ in the special case of a first order operator. Throughout this section we set $s=1$ and, without loss of generality, we assume $m^{+} \geq 1$.

Let $v_{k}$ be a sequence of orthonormalised eigenfunctions of the operator $A$ corresponding to the sequence of eigenvalues (3.8). Let $\widetilde{v}_{k}$ be a sequence of orthonormalised eigenfunctions of the operator $\widetilde{A}$ corresponding to the sequence of eigenvalues (3.12).

Let us define the spectral densities

$$
N(x, \lambda):= \begin{cases}0 & \text { if } \lambda \leq 0, \\ \sum_{k: 0<\lambda_{k}<\lambda}\left[v_{k}(x)\right]^{*} v_{k}(x) & \text { if } \lambda>0,\end{cases}
$$

and

$$
\widetilde{N}(x, \lambda):= \begin{cases}0 & \text { if } \lambda \leq 0, \\ \sum_{k: 0<\zeta_{k}<\lambda}\left[\widetilde{v}_{k}(x)\right]^{*} \widetilde{v}_{k}(x) & \text { if } \lambda>0 .\end{cases}
$$

The quantities (5.33) and (5.34) are sometimes referred to as local counting functions, because they count the number of positive eigenvalues of the operator below a given $\lambda$, with $x$-dependent (hence 'local') weight given by the modulus squared of the corresponding eigenfunctions.

Integrating (5.33) and (5.34) one obtains the usual (global positive) counting functions

$$
N(\lambda):=\int_{M} N(x, \lambda) d x= \begin{cases}0 & \text { if } \lambda \leq 0 \\ \sum_{k: 0<\lambda_{k}<\lambda} 1 & \text { if } \lambda>0\end{cases}
$$

and

$$
\widetilde{N}(\lambda):=\int_{M} \tilde{N}(x, \lambda) d x= \begin{cases}0 & \text { if } \lambda \leq 0 \\ \sum_{k: 0<\zeta_{k}<\lambda} 1 & \text { if } \lambda>0 .\end{cases}
$$

Note that (5.33) and (5.34) are densities over $M$, whereas (5.35) and (5.36) are scalar functions.

For each $j \in J$ let

$$
t \mapsto\left(x^{(j)}(t ; y, \eta), \xi^{(j)}(t ; y, \eta)\right)
$$

be the solution to Hamilton's equations

$$
\dot{x}^{(j)}=h_{\xi}^{(j)}\left(x^{(j)}, \xi^{(j)}\right), \quad \dot{\xi}^{(j)}=-h_{x}^{(j)}\left(x^{(j)}, \xi^{(j)}\right)
$$

with initial condition $\left(x^{(j)}(0 ; y, \eta), \xi^{(j)}(0 ; y, \eta)\right)=(y, \eta) \in T^{\prime} M$. Define $T_{0}$ to be the infimum of lengths of all the Hamiltonian loops generated by the Hamiltonian flow (5.37) for all $j \in J$ and all possible initial conditions. Let $\hat{\mu}: \mathbb{R} \rightarrow \mathbb{C}$ be a smooth function such that $\mu \equiv 1$ in a neighbourhood of 0 and $\operatorname{supp} \hat{\mu} \subset\left(-T_{0}, T_{0}\right)$. Let $\mu:=\mathcal{F}_{t \rightarrow \lambda}^{-1} \hat{\mu}$ be the inverse Fourier transform of $\hat{\mu}$. 
It is well known $[20,31,32,33,46,47,48]$ that the mollified derivative of the spectral density admits a complete asymptotic expansion in integers powers of $\lambda$ :

$$
\begin{aligned}
& \left(N^{\prime} * \mu\right)(x, \lambda)=w_{d-1}(x) \lambda^{d-1}+w_{d-2}(x) \lambda^{d-2}+\ldots \quad \text { as } \quad \lambda \rightarrow+\infty, \\
& \left(\widetilde{N}^{\prime} * \mu\right)(x, \lambda)=\widetilde{w}_{d-1}(x) \lambda^{d-1}+\widetilde{w}_{d-2}(x) \lambda^{d-2}+\ldots \quad \text { as } \quad \lambda \rightarrow+\infty .
\end{aligned}
$$

Here the prime denotes the derivative in $\lambda$ and $*$ convolution with respect to $\lambda$. The smooth densities appearing as coefficients of powers of $\lambda$ in (5.38) and (5.38) are usually called local Weyl coefficients. Integrating the local Weyl coefficients over $M$ one obtains scalars known as (global) Weyl coefficients.

Computing Weyl coefficients is a delicate task. A formula for the second local Weyl coefficient $w_{d-2}$ for systems $(m \geq 2)$ was first obtained in 2013 by Chervova, Downes and Vassiliev [15], fixing decades of incorrect or incomplete publications in the subject (see [15, Section 11]), and later recovered by a completely different method by Avetisyan, Sjöstrand and Vassiliev [1]. The formula for $w_{d-2}$ reads [15, Eqn (1.24)] [1, Eqn. (1.6)] [14, Eqn. (5.10)]

$$
\begin{aligned}
& w_{d-2}^{(j)}(x)=-\frac{d(d-1)}{(2 \pi)^{d}} \int_{h^{(j)}(x, \xi)<1} \operatorname{tr}\left(P^{(j)} A_{\mathrm{sub}}+\frac{i}{2}\left\{P^{(j)}, P^{(j)}\right\} A_{\text {prin }}\right. \\
& \left.-\frac{1}{d-1} h^{(j)}\left(P_{j}\right)_{\mathrm{sub}}\right)(x, \xi) d \xi .
\end{aligned}
$$

Working with systems, as opposed to scalar equations, introduces substantial difficulties, which one needs to overcome and which are simply not present when studying the spectrum of scalar problems.

Now, the diagonalization argument presented above effectively reduces our original system $A$ to a collection of scalar operators, the diagonal operator $\widetilde{A}$. It is then natural to ask whether the approximate diagonalization offers a way to provide a different proof for (5.40), one that relies on the corresponding formula for scalar operators. Indeed, it is easy to see that the unique (modulo $\Psi_{m}^{-\infty}$ ) pseudodifferential basis given by Theorem 2.4 associated with the operator $\widetilde{A}$ is

$$
\widetilde{P}_{j}:=B^{*} P_{j} B=\widetilde{v}^{(j)}\left[\widetilde{v}^{(j)}\right]^{*} \operatorname{Id} \quad \bmod \Psi^{-\infty}, \quad j \in J,
$$

where the $\widetilde{v}^{(j)}$,

$$
\left[\widetilde{v}^{(j)}\right]_{q}=\delta_{j q}, \quad j, q \in J
$$

are the eigenvectors of $\widetilde{A}_{\text {prin }}$. Formula (5.41) immediately implies

$$
\left(\widetilde{P}_{j}\right)_{\mathrm{sub}}=0, \quad j \in J .
$$

On account of (5.42) and (5.43), formula (5.40) applied to the operator $\widetilde{A}$ gives us

$$
\widetilde{w}_{d-2}^{(j)}(x)=-\frac{d(d-1)}{(2 \pi)^{d}} \sum_{j=1}^{m^{+}} \int_{h^{(j)}(x, \xi)<1}\left(a_{j}\right)_{\mathrm{sub}}(x, \xi) d \xi,
$$


namely, as expected, the second Weyl coefficient for $\widetilde{A}$ is the sum of the second Weyl coefficients of the scalar operators $a_{j}, j=1, \ldots, m^{+}$, computed in accordance with the classical Duistermaat and Guillemin's formula for scalar elliptic operators [20, Eqn. (2.2)].

Unfortunately, this doesn't quite work: the operators $A$ and $\widetilde{A}$ are related via conjugation by the pseudodifferential operator $B$. Even though, as we have shown, this does not change the spectrum of $A$ too much - where 'not too much' is quantified by the remainder term in (3.13) - there is no reason why it should preserve the asymptotic expansion of the spectral density (5.33). The diagonalization argument as presented in this paper only allows one to recover global spectral asymptotics. In other words, one can exploit diagonalization to recover in a simpler way - i.e. relying only on formulae for scalar operators - the integral over $M$ of (5.40), but not (5.40) itself.

To shed additional light onto the above discussion, let us compare the approach pursued in this paper with that of [14]. In [14] the author and Vassiliev showed that the spectrum of an elliptic system $A$ partitions into $m$ series of eigenvalues, labelled by the eigenvalues $h^{(j)}$ of $A_{\text {prin }}$, up to a superpolynomial error. This mirrors, at the spectral level, the decomposition of $L^{2}(M)$ into almost-orthogonal almost-invariant subspaces via the pseudodifferential projections $P_{j}$, see Theorem 2.4. This result was achieved by showing that the positive spectrum of $A$ is approximated by the union of the positive spectra of (a minor modification of) the elliptic matrix operators $A_{j}, j=1, \ldots, m^{+}$, defined in accordance with (5.7). For a first order system, it was also shown that the positive part of the propagator $U(t):=e^{-i t A}$ decomposes, in a similar manner, as

$$
\theta(A) U(t)=\sum_{j=1}^{m^{+}} U^{(j)}(t) \quad \bmod C^{\infty}\left(\mathbb{R} ; \Psi_{m}^{-\infty}\right),
$$

where

$$
U^{(j)}(t)=P_{j} U(t)=U(t) P_{j}=\theta\left(A_{j}\right) e^{-i t A_{j}} \quad \bmod C^{\infty}\left(\mathbb{R} ; \Psi_{m}^{-\infty}\right), \quad j=1, \ldots, m^{+} .
$$

Here $\theta(\cdot)$ is the Heaviside theta function, see [13, Sec. 7]. We refer the reader to $[15,9,12,7]$ for additional details on $U(t), U^{(j)}(t)$ and their explicit construction. Properties (5.44) and (5.45) allowed us to use Levitan's wave method to compute local asymptotics for the spectral density 'along invariant subspaces' and express the second local Weyl coefficients of $A$ as the sum of the second local Weyl coefficients of the $A_{j}$ 's.

The same argument wouldn't work when approaching the problem via diagonalization. Indeed, let $\widetilde{U}(t):=e^{-i t \widetilde{A}}$. Using (5.44), (5.45) and (3.3), it is not difficult to see that

$$
B^{*} U^{(j)}(t) B=\widetilde{P}^{(j)} e^{-i t a_{j}} \bmod C^{\infty}\left(\mathbb{R} ; \Psi_{m}^{-\infty}\right)
$$

and

$$
\theta(A) U(t)=B \theta(\widetilde{A}) e^{-i t \widetilde{A}} B^{*}=B\left(\sum_{j=1}^{m^{+}} \widetilde{P}^{(j)} e^{-i t a_{j}}\right) B^{*} \bmod C^{\infty}\left(\mathbb{R} ; \Psi_{m}^{-\infty}\right)
$$


where $\widetilde{P}^{(j)}:=\widetilde{v}^{(j)}\left[\widetilde{v}^{(j)}\right]^{*}($ recall $(5.42))$. In (5.46) and (5.47), unlike in (5.45), the propagator of $A$ and that of $\widetilde{A}$ are not related directly, but via conjugation by the pseudodifferential operator $B$.

All in all, on the one hand the diagonalization approach presented here has the advantage of turning an elliptic system into a collection of elliptic scalar operators in a way that essentially preserves the spectrum and is compatible with pseudodifferential projections $P_{j}$, which may be quite useful when dealing with applications, say, to operators of interest in theoretical physics. On the other hand, the approach pursued in [14] is, in a sense, more natural to study the spectrum of $A$, in that it captures finer information, and it always works, even in the presence of the topological obstructions from Remark 2.2.

\section{An example: the operator of linear elasticity}

In this section we apply our results to an explicit example: the Lamé operator in dimension 2. Throughout this section we adopt Einstein's summation convention over repeated indices.

Let $M$ be the 2-torus $\mathbb{T}^{2}$ endowed with the standard flat metric $g$. The operator of linear elasticity $L$ on vector fields, also known as the Lamé operator, is defined in accordance with

$$
\frac{1}{2} \int_{M} g_{\alpha \beta} v^{\alpha}(L v)^{\beta} \rho d x=E(v),
$$

where $E(v)$ is the potential energy of elastic deformation

$$
E(v):=\frac{1}{2} \int_{M}\left(\lambda\left(\nabla_{\alpha} v^{\alpha}\right)^{2}+\mu\left(\nabla_{\alpha} v_{\beta}+\nabla_{\beta} v_{\alpha}\right) \nabla^{\alpha} v^{\beta}\right) \rho d x,
$$

$\rho(x):=\sqrt{\operatorname{det} g_{\alpha \beta}(x)}$ is the Riemannian density, $\nabla$ is the Levi-Civita connection, and the scalars $\lambda$ and $\mu$ are the Lamé parameters. We refer the reader to $[35,11]$ for further details on the derivation. In order to guarantee strong convexity, the Lamé parameters are assumed to satisfy the conditions

$$
\mu>0, \quad \lambda+\mu>0,
$$

see, e.g., [38]. Integrating (6.1) by parts yields an explicit formula for $L$ :

$$
(L v)^{\alpha}=-\mu\left(\nabla_{\beta} \nabla^{\beta} v^{\alpha}+\operatorname{Ric}_{\beta}^{\alpha} v^{\beta}\right)-(\lambda+\mu) \nabla^{\alpha} \nabla_{\beta} v^{\beta} .
$$

Here Ric is the Ricci tensor.

In order to apply our results, we need to turn the operator $L$, which acts on 2-vectors, into an operator acting on 2-columns of half-densities. This is done as follows.

Let $e_{j}, j=1,2$, be a global orthonormal framing on $\mathbb{T}^{2}$; we denote by $e_{j}{ }^{\alpha}$ the $\alpha$-th component of the $j$-th vector field. Put

$$
e_{\alpha}^{j}:=\delta^{j k} g_{\alpha \beta} e_{k}^{\beta},
$$

and consider the operator $S$,

$$
(S v)^{j}:=e_{\alpha}^{j} v^{\alpha},
$$


which maps 2-vectors to 2-columns of scalar functions. The operator of linear elasticity acting on half-densities is defined as

$$
L_{1 / 2}:=\rho^{1 / 2} S L S^{-1} \rho^{-1 / 2} .
$$

Of course, $L_{1 / 2} \in \Psi_{2}^{2}$.

In what follows we will compute the subprincipal symbol of the approximate diagonalization of $L_{1 / 2}$. In order to express our formulae in terms of geometric invariants, we need to introduce some additional definitions.

Let $\nabla_{W}$ be the Weitzenböck connection associated with the framing $\left\{e_{j}\right\}_{j=1}^{2}$, namely, the curvature-free metric compatible connection whose connection coefficients are

$$
\Upsilon_{\beta \gamma}^{\alpha}:=e_{j}{ }^{\alpha} \frac{\partial e_{\gamma}^{j}}{\partial x^{\beta}} .
$$

As we are working in dimension two, the torsion tensor $T_{\beta \gamma}^{\alpha}:=\Upsilon^{\alpha}{ }_{\beta \gamma}-\Upsilon^{\alpha}{ }_{\gamma \beta}$ of $\nabla_{W}$ is equivalent to a covector field

$$
t_{\alpha}:=\frac{1}{2} T_{\alpha}^{\beta \gamma} \epsilon_{\beta \gamma} \rho,
$$

where $\epsilon_{\beta \gamma}$ is the totally antisymmetric symbol, $\epsilon_{12}=+1$. Here and further on, indices are raised and lowered using the metric, via the musical isomorphism.

By means of straightforward calculations, formulae (6.3)-(6.6) yield

$$
\left(L_{1 / 2}\right)_{\text {prin }}=\mu h^{2} I+(\lambda+\mu) h^{2} p p^{T}
$$

and

$$
\left(L_{1 / 2}\right)_{\mathrm{sub}}=i(\lambda+3 \mu) t^{\alpha} \xi_{\alpha} \epsilon,
$$

where

$$
h(x, \xi):=\sqrt{g^{\alpha} \beta(x) \xi_{\alpha} \xi_{\beta}}, \quad p:=\frac{1}{h}\left(\begin{array}{c}
e_{1}^{\alpha} \xi_{\alpha} \\
e_{2}^{\alpha} \xi_{\alpha}
\end{array}\right), \quad \epsilon:=\left(\begin{array}{cc}
0 & 1 \\
-1 & 0
\end{array}\right) .
$$

Analysing (6.7) we conclude that the eigenvalues of $\left(L_{1 / 2}\right)_{\text {prin }}$ are

$$
h^{(1)}=\mu h^{2}, \quad h^{(2)}=(\lambda+2 \mu) h^{2}
$$

and the corresponding orthonormalised eigenvectors are

$$
v^{(1)}=\epsilon p, \quad v^{(2)}=p .
$$

Note that conditions (6.2) imply $h^{(2)} / h^{(1)}>1$, so, in particular, that the eigenvalues are simple.

Theorem 6.1. Let $\widetilde{L}_{1 / 2}:=B^{*} L_{1 / 2} B$, where $B$ is the almost-unitary operator given by Theorems 3.1 and 3.3 for $A=L_{1 / 2}$. Then we have

$$
\left(\widetilde{L}_{1 / 2}\right)_{\mathrm{sub}}=0 .
$$


Proof. Proving (6.12) is equivalent to showing that

$$
\left(B_{j}^{*} L_{1 / 2} B_{j}\right)_{\mathrm{sub}}=0, \quad j=1,2 .
$$

Let us begin by computing $\left(B_{j}\right)_{\mathrm{sub}}, j=1,2$. It was shown in [13] that

$$
\left(P_{j}\right)_{\mathrm{sub}}=0, \quad j=1,2 .
$$

Furthermore, it is not hard to see that

$$
\left\{P^{(1)}, v^{(1)}\right\}=\epsilon\left\{P^{(2)}, v^{(2)}\right\}=0 .
$$

On account of (6.14) and (6.15), Theorem 3.2 gives us

$$
\left(B_{j}\right)_{\mathrm{sub}}=i f^{(j)} v^{(j)}, \quad j=1,2 .
$$

Of course,

$$
\left(B_{j}^{*}\right)_{\mathrm{sub}}=\left[\left(B_{j}\right)_{\mathrm{sub}}\right]^{*}=-i f^{(j)}\left[v^{(j)}\right]^{*} .
$$

Now, applying (5.6) to $B_{j}^{*} L_{1 / 2} B_{j}$ and using (6.16), (6.17) we obtain

$$
\begin{aligned}
\left(B_{j}^{*} L_{1 / 2} B_{j}\right)_{\mathrm{sub}} & =\left[v^{(j)}\right]^{*}\left(L_{1 / 2}\right)_{\mathrm{sub}} v^{(j)}+\frac{i}{2}\left\{\left[v^{(j)}\right]^{*},\left(L_{1 / 2}\right)_{\operatorname{prin}}, v^{(j)}\right\} \\
& +\frac{i}{2}\left(\left[v^{(j)}\right]^{*}\left\{\left(L_{1 / 2}\right)_{\operatorname{prin}}, v^{(j)}\right\}+\left\{\left[v^{(j)}\right]^{*},\left(L_{1 / 2}\right)_{\operatorname{prin}}\right\} v^{(j)}\right) .
\end{aligned}
$$

Formulae (6.8), (6.9) and (6.11) imply

$$
\left[v^{(j)}\right]^{*}\left(L_{1 / 2}\right)_{\mathrm{sub}} v^{(j)}=0, \quad j=1,2 .
$$

The Spectral Theorem tells us that

$$
\left(L_{1 / 2}\right)_{\text {prin }}=h^{2}\left(\mu I+(\lambda+\mu) P^{(2)}\right)=(\lambda+2 \mu) I-(\lambda+\mu) P^{(1)} .
$$

The latter, combined with the identities

$$
\left\{\left[v^{(j)}\right]^{*}, v^{(j)}\right\}=0, \quad\left\{\left[v^{(j)}\right]^{*}, P^{(j)}, v^{(j)}\right\}=0, \quad j=1,2,
$$

in turn gives us

$$
\left\{\left[v^{(j)}\right]^{*},\left(L_{1 / 2}\right)_{\operatorname{prin}}, v^{(j)}\right\}=0, \quad j=1,2 .
$$

Finally, we observe that

$$
\left\{\left(L_{1 / 2}\right)_{\operatorname{prin}}, v^{(j)}\right\}=-\left\{\left[v^{(j)}\right]^{*},\left(L_{1 / 2}\right)_{\operatorname{prin}}\right\}^{*}
$$

and

$$
\left\{\left[v^{(j)}\right]^{*},\left(L_{1 / 2}\right)_{\operatorname{prin}}\right\} v^{(j)}=\left(\left\{\left[v^{(j)}\right]^{*},\left(L_{1 / 2}\right)_{\operatorname{prin}}\right\} v^{(j)}\right)^{*},
$$

so that

$$
\left[v^{(j)}\right]^{*}\left\{\left(L_{1 / 2}\right)_{\operatorname{prin}}, v^{(j)}\right\}+\left\{\left[v^{(j)}\right]^{*},\left(L_{1 / 2}\right)_{\operatorname{prin}}\right\} v^{(j)}=0, \quad j=1,2 .
$$

Substituting (6.19), (6.20) and (6.21) into (6.18) we arrive at (6.13).

Theorem 6.1 implies that the second (global) Weyl coefficient for the operator of linear elasticity in dimension 2 vanishes, which agrees with known results [48, Chapter 6]. 


\section{Acknowledgements}

I am grateful to Alex Strohmaier for raising questions that eventually led to this paper and for pointing out useful references; to Jean-Claude Cuenin for helpful comments on a preliminary version of this manuscript and for bibliographic suggestions; to Grigori Rozenbloum for insightful discussions on the role of topological obstructions; to Dmitri Vassiliev for numerous discussions on this and related topics throughout the years.

This work was supported by the Leverhulme Trust Research Project Grant RPG-2019240, which is gratefully acknowledged.

\section{Appendix A Some useful results}

We collect in this appendix, for the reader's convenience, a few results from [14] that are used in the main text of the paper.

Lemma A.1 ([14, Lemma 3.6]). Let $A \in \Psi_{m}^{s}, s>0$, be an operator as in Section 2. Let $\mu_{1} \leq \mu_{2} \leq \cdots \leq \mu_{r}$ be real numbers and let $u_{k}, k=1, \ldots, r$, be an orthonormal set in $L^{2}(M)$. Suppose that

$$
\left\|\left(A-\mu_{k}\right) u_{k}\right\|_{L^{2}} \leq \varepsilon, \quad k=1, \ldots, r .
$$

Then

$$
\#\left\{\lambda \mid \lambda \in \sigma(A) \cap\left[\mu_{1}-\sqrt{r} \varepsilon, \mu_{r}+\sqrt{r} \varepsilon\right]\right\} \geq r .
$$

Lemma A.2 ([14, Lemma 3.9]). Let $F$ be an Hermitian $r \times r$ matrix such that

$$
\|F-I\|_{\max } \leq \frac{1}{3 r^{2}}
$$

where $I$ is the $r \times r$ identity matrix and $\|F\|_{\max }:=\max _{1 \leq j, k \leq r}\left|F_{j k}\right|$ is the max matrix norm. Then there exists an Hermitian matrix $G$ such that

$$
G F G=I
$$

and

$$
\|G-I\|_{\max } \leq\|F-I\|_{\max }
$$

Let $A \in \Psi_{m}^{s}, s>0$, be an operator as in Section 2 and let

$$
N(\lambda):= \begin{cases}0 & \text { for } \lambda \leq 0 \\ \sum_{k: 0<\lambda_{k}<\lambda} 1 & \text { for } \lambda>0\end{cases}
$$

be its (positive) counting function.

Theorem A.3 (Weyl's law for elliptic systems [14, Theorem B.1]). We have

$$
N(\lambda)=b \lambda^{d / s}+o\left(\lambda^{d / s}\right) \text { as } \lambda \rightarrow+\infty,
$$

where

$$
b=\frac{1}{(2 \pi)^{d}} \sum_{j=1}^{m^{+}} \int_{h^{(j)}(x, \xi)<1} \mathrm{dVol}_{T^{*} M} .
$$




\section{References}

[1] Z. Avetisyan, J. Sjöstrand and D. Vassiliev, The second Weyl coefficient for a first order system, in: Analysis as a tool in mathematical physics, P. Kurasov, A. Laptev, S. Naboko and B. Simon (Eds.), Operator Theory: Advances and Applications 276 Birkhäuser Verlag (2020) 120-153.

[2] V. Bach and J.-B. Bru, Rigorous foundations of the Brockett-Wegner flow for operators, J. Evol. Equ. 10 (2010) 425-442.

[3] E. I. Blount, Extension of the Foldy-Wouthuysen Transformation, Phys. Rev. 128 (1962) 2454-2458.

[4] J. Bolte and R. Glaser, Semiclassical Egorov theorem and quantum ergodicity for matrix valued operators, Comm. Math. Phys. 247 (2004) 391-419.

[5] R. Brummelhuis and J. Nourrigat, Scattering amplitude for dirac operators, Comm. Partial Differential Equations 24 no. 1-2 (1999) 377-394.

[6] V. Bruneau and D. Robert, Asymptotics of the scattering phase for the Dirac operator: High energy, semi-classical and non-relativistic limits, Ark. Mat. 37 (1999) 1-32.

[7] M. Capoferri, C. Dappiaggi and N. Drago, Global wave parametrices on globally hyperbolic spacetimes, J. Math. Anal. Appl. 490 (2020) 124316.

[8] M. Capoferri, G. Rozenbloum, N. Saveliev and D. Vassiliev, Topological obstructions to the diagonalization of elliptic systems. In preparation.

[9] M. Capoferri, M. Levitin and D. Vassiliev, Geometric wave propagator on Riemannian manifolds. Preprint arXiv:1902.06982 (2019), to appear in Comm. Anal. Geom.

[10] M. Capoferri, N. Saveliev and D. Vassiliev, Classification of first order sesquilinear forms, Rev. Math. Phys. 32 (2020) 2050027.

[11] M. Capoferri and D. Vassiliev, Spacetime diffeomorphisms as matter fields, J. Math. Phys. 61 (2020) 111508.

[12] M. Capoferri and D. Vassiliev, Global propagator for the massless Dirac operator and spectral asymptotics. Preprint arXiv:2004.06351 (2020).

[13] M. Capoferri and D. Vassiliev, Invariant subspaces of elliptic systems I: pseudodifferential projections. Preprint arXiv:2103.14325 (2021).

[14] M. Capoferri and D. Vassiliev, Invariant subspaces of elliptic systems II: Spectral theory, J. Spectr. Theory, to appear.

[15] O. Chervova, R. J. Downes and D. Vassiliev, The spectral function of a first order elliptic system, J. Spectr. Theory 3 no. 3 (2013) 317-360. 
[16] H. O. Cordes, A version of Egorov's theorem for systems of hyperbolic pseudo-differential equations, J. Funct. Anal. 48 no. 3 (1982) 285-300.

[17] H. O. Cordes, A pseudodifferential-Foldy-Wouthuysen transform, Comm. Partial Differential Equations 8 (1983) 1475-1485.

[18] H. O. Cordes, A precise pseudodifferential Foldy-Wouthuysen transform for the Dirac equation, J. Evol. Equ. 4 (2004) 125-138.

[19] J.-C. Cuenin, Block-diagonalization of operators with gaps, with applications to Dirac operators, Rev. Math. Phys. 24 no. 8 (2012) 1250021.

[20] J. J. Duistermaat and V. W. Guillemin, The spectrum of positive elliptic operators and periodic bicharacteristics, Invent. Math. 29 no. 1 (1975) 39-79.

[21] J. J. Duistermaat and L. Hörmander, Fourier integral operators. II., Acta Math. 128 no. 3-4 (1972) 183-269.

[22] Z. Avetisyan, Y.-L. Fang, N. Saveliev and D. Vassiliev, Analytic definition of spin structure, J. Math. Phys. 58 no. 8 (2017) 082301.

[23] L. L. Foldy, The electromagnetic properties of the Dirac particles, Phys. Rev. 87 no. 5 (1952) 688-693.

[24] L. L. Foldy and S. A. Wouthuysen, On the Dirac theory of spin $1 / 2$ particles and its non-relativistic limit, Phys. Rev. 78 no. 1 (1950) 29-36.

[25] C. Gérard and M. Wrochna, Analytic Hadamard states, Calderón projectors and Wick rotation near analytic Cauchy surfaces, Comm. Math. Phys. 366 (2019) 29-65.

[26] C. Gérard and M. Wrochna, The massive Feynman propagator on asymptotically Minkowski spacetimes, Amer. J. Math. 141 no. 6 (2019) 1501-1546.

[27] C. Gérard and M. Wrochna, The massive Feynman propagator on asymptotically Minkowski spacetimes II, Int. Math. Res. Not. 2020 no. 20 (2020) 6856-6870.

[28] H. Langer and C. Tretter, Diagonalization of certain block operator matrices and applications to Dirac operators. In: Operator Theory and Analysis, H. Bart, A. C. M. Ran and I. Gohberg (Eds.), Oper. Theory Adv. Appl. 122, Birkhäuser, Basel (2001) 331-358.

[29] B. Helffer and J. Sjöstrand, Analyse semi-classique pour l'équation de Harper. II : comportement semi-classique près d'un rationnel, Mém. Soc. Math. Fr. 40 (1990).

[30] B. A. Hess, M. Reiher and A. Wolf, The generalized Douglas-Kroll transformation, J. Chem. Phys. 117 no. 20 (2002) 9215-9226.

[31] V. Ivrii, Second term of the spectral asymptotic expansion of the Laplace-Beltrami operator on manifolds with boundary, Funct. Anal. Appl. 14 (1980) 98-106. 
[32] V. Ivrii, Precise spectral asymptotics for elliptic operators acting in fiberings over manifolds with boundary, Lecture Notes in Mathematics 1100, Springer-Verlag, Berlin, 1984.

[33] V. Ivrii, Microlocal analysis and precise spectral asymptotics, Springer-Verlag, Berlin, 1998.

[34] J. Lagacé, S. Morozov, L. Parnovski, B. Pfirsch and R. Shterenberg, The almost periodic gauge transform - An abstract scheme with applications to Dirac Operators. Preprint arXiv:2106.01888 (2021).

[35] L. D. Landau and E. M. Lifshitz, Theory of elasticity, course of theoretical physics vol 7, 3rd edn (Pergamon, Oxford, 1986). Translated from the Russian by J. B. Sykes and W. H. Reid.

[36] R. G. Littlejohn and W. G. Flynn, Geometric phases in the asymptotic theory of coupled wave equations, Phys. Rev. A 44 (1991) 5239-5256.

[37] R. G. Littlejohn and S. Weigert, Diagonalization of multicomponent wave equations with a Born-Oppenheimer example, Phys. Rev. A 47 (1993) 3506-3512.

[38] Y. Miyanishi and G. Rozenblum, Spectral properties of the Neumann-Poincaré operator in 3D elasticity, Int. Math. Res. Not. 2021 no. 11 (2021) 8715-8740.

[39] G. Nenciu and V. Sordoni, Semiclassical limit for multistate Klein-Gordon systems: almost invariant subspaces and scattering theory, J. Math. Phys. 45 (2004) 3676.

[40] W. J. Nicoll, Global oscillatory integrals for solutions of hyperbolic systems, $\mathrm{PhD}$ thesis, University of Sussex (1998).

[41] G. Panati, H. Spohn and S. Teufel, Space-adiabatic perturbation theory, Adv. Theor. Math. Phys. 7 (2003) 145-204.

[42] M. Reiher and A. Wolf, Exact decoupling of the Dirac Hamiltonian. I. General theory, J. Chem. Phys. 121 no. 5 (2004) 2037-2047.

[43] M. Reiher and A. Wolf, Exact decoupling of the Dirac Hamiltonian. II. The generalized Douglas-Kroll-Hess transformation up to arbitrary order, J. Chem. Phys. 121 no. 22 (2004) 10945-10956.

[44] M. Reiher and A. Wolf, Exact decoupling of the Dirac Hamiltonian. III. Molecular properties, J. Chem. Phys. 124 no. 6 (2006) 064102.

[45] M. Reiher and A. Wolf, Exact decoupling of the Dirac Hamiltonian. IV. Automated evaluation of molecular properties within the Douglas-Kroll-Hess theory up to arbitrary order, J. Chem. Phys. 124 no. 6 (2006) 064103.

[46] G. Rozenblyum, Spectral asymptotic behavior of elliptic systems, J. Math. Sci. 21 (1983) 837-850. 
[47] Yu. Safarov, Non-classical two-term spectral asymptotics for self-adjoint elliptic operators. DSc thesis, Leningrad Branch of the Steklov Mathematical Institute of the USSR Academy of Sciences (1989). In Russian.

[48] Yu. Safarov and D. Vassiliev, The asymptotic distribution of eigenvalues of partial differential operators, Amer. Math. Soc., Providence (RI), 1997.

[49] D. Shen and M. Wrochna, An index theorem on asymptotically static spacetimes with compact Cauchy surface. Preprint arXiv:2104.02816 (2021).

[50] H. Siedentop and E. Stockmeyer, The Douglas-Kroll-Heß method: convergence and block-diagonalization of Dirac operators, Ann. Henri Poincaré 7 no. 1 (2006) 45-58.

[51] M. E. Taylor, Reflection of singularities of solutions of systems of differential equations, Comm. Pure Appl. Math. 28 (1975) 457-478.

[52] F. Wegner, Flow equations for Hamiltonians, Ann. Phys. 3 (1994) 77-91. 